\title{
Stratification of venous thromboembolism risk in stroke patients by Caprini score
}

\author{
Xiaoping Zhu' ${ }^{1}$, Tingting Zhang ${ }^{2}$, Lanshu Zhou ${ }^{1}$, Xiaobing Yin ${ }^{2}$, Qiong Dong ${ }^{2}$ \\ ${ }^{1}$ School of Nursing, Second Military Medical University, Shanghai 200433, China; ${ }^{2}$ Shanghai Tenth People’s Hospital, Tongji University, Shanghai \\ 200072, China \\ Contributions: (I) Conception and design: X Zhu, L Zhou; (II) Administrative support: L Zhou; (III) Provision of study material or patients: All \\ authors; (IV) Collection and assembly of data: All authors; (V) Data analysis and interpretation: X Zhu; (VI) Manuscript writing: All authors; (VII) \\ Final approval of manuscript: All authors. \\ Correspondence to: Lanshu Zhou. School of Nursing, Second Military Medical University, No. 800 Xiangyin Road, Yangpu District, Shanghai 200433, \\ China. Email: zhoulanshu@hotmail.com.
}

\begin{abstract}
Background: Venous thromboembolism (VTE) includes deep vein thrombosis (DVT) and pulmonary embolus (PE) is a serious complication in hospitalized stroke patients. This study is designed to examine the incidence of VTE and the effectiveness of chemo thromboprophylaxis in this population.

Methods: From January 2015 to April 2018, we calculate the Caprini score for every admitted patient to evaluate VTE risk. For analysis Demographic information, Caprini score, national institutes of health stroke scale (NIHSS) score, prothrombin time (PT), thrombin time (TT), D-Dimer and medical co-morbidity are noted.

Results: Of 3,824 inpatients during the study period, 21 patients $(0.55 \%)$ have VTE complications. Among patients $(\mathrm{n}=148)$ with a Caprini score between 0 and 2,1 patient $(0.68 \%)$ have VTE. VTE $(52.38 \%)$ most commonly occurred in patients of Caprini score 7-8 group. In patients with VTE $(n=21)$ age, Caprini score, NIHSS score, National Early Warning Score (NEWS) score, PT, TT, D-Dimer are significantly higher than those (n=3,803) without VTE $(\mathrm{P}<0.05)$.

Conclusions: The Caprini score is highly recommended to assess the risk of venous thrombosis in stroke patients. Early mechanical and chemical prophylaxis are recommended for patients to reduce the incidence of venous thrombosis.
\end{abstract}

Keywords: Stroke; Caprini score; venous thromboembolism (VTE)

Submitted Jun 16, 2019. Accepted for publication Feb 10, 2020.

doi: 10.21037/apm.2020.04.20

View this article at: http://dx.doi.org/10.21037/apm.2020.04.20

\section{Introduction}

Venous thromboembolism (VTE) is a common complication after an ischemic stroke that includes deep vein thrombosis (DVT) and pulmonary embolism (PE) and their risk is obviously higher in stroke patients than others. It contributes to extend hospitalized period which results in increased expense $(1,2)$. To reduce the risk of VTE we can administer chemoprophylaxis, but there are still some side effects of anticoagulants, for instance, the risk of bleeding and coagulopathy (3). An urgent risk assortment is necessary for physician to optimize thromboprophylaxis regimens.
The Caprini RAM (4) is the most widely used and guideline recommended tool for VTE risk prediction, also wellvalidated in stroke patients. Since the occurrence of VTE in stroke patients is poorly characterized, we conducted this retrospective study to (I) investigate the incidence of symptomatic VTE; (II) examine the utility of the Caprini RAM for VTE risk stratification.

\section{Methods}

This is a retrospective study conducted in Shanghai 


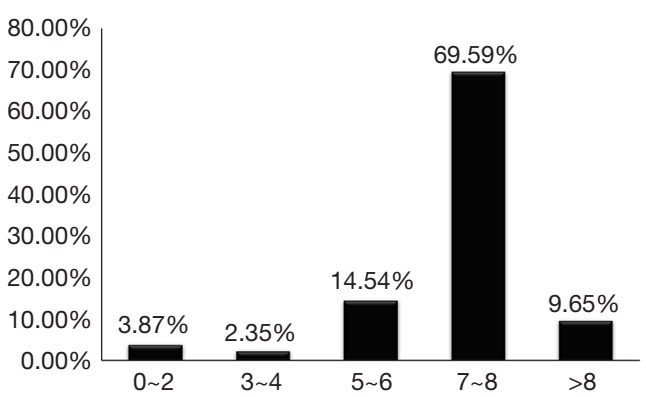

Figure 1 Distributions of patients according to the Caprini score, $\mathrm{n}=3,824$.

Table 1 Demographic and clinical characteristics of stroke patients

\begin{tabular}{lc}
\hline Characteristics & Patients \\
\hline Gender, n (\%) & \\
Male & $2,510(65.6)$ \\
Female & $1,314(34.4)$ \\
Age (years), mean \pm SD & $68.27 \pm 11.33$ \\
Medical co-morbidity, n (\%) & \\
Hypertension & $3,077(80.5)$ \\
Atrial fibrillation & $361(9.40)$ \\
Coronary heart disease & $363(9.50)$ \\
Diabetes & $1,509(39.5)$ \\
Cancer & $50(1.30)$ \\
Hyperbaric therapy & $676(17.7)$ \\
NIHSS score, mean \pm SD & $3.39 \pm 3.71$ \\
NEWS score, mean \pm SD & $1.54 \pm 1.12$ \\
PT, mean \pm SD & $11.62 \pm 1.33$ \\
APTT, mean \pm SD & $27.95 \pm 4.88$ \\
TT, mean \pm SD & $19.89 \pm 5.13$ \\
D-dimer, mean \pm SD & $0.85 \pm 1.88$ \\
Day of anticoagulants, mean \pm SD & $6.40 \pm 2.65$
\end{tabular}

NIHSS, National Institutes of Health Stroke Scale; NEWS, National Early Warning Score; PT, prothrombin time; APTT, activated partial thromboplastin time; TT, thrombin time; SD, standard deviation.

Tenth People's Hospital of Tongji University. The study is approved by National Ethics Committee of Shanghai Tenth People's Hospital of Tongji University (No. 2019-k51). All the patients provided the written informed consent.
After approval of ethical committee, data is collected from hospital record. Inclusion criteria: all the patient admitted to Neurology Department between January 1,2015 to April 31,2018 are included. Exclusion criteria: all pediatric patients are excluded. In total 3,824 patients (1,314 female and 2,510 male) with mean age of $68.77 \pm 11.33$ years are included in the study. Data regarding demographics, concomitant diseases, National Institutes of Health Stroke Scale (NIHSS) score, National Early Warning Score (NEWS) score, Caprini score, thromboprophylaxis and laboratory indexes are collected from the electronic medical record system. If there are no contraindication then mechanical prophylaxis (graduated compression stockings) is recommended as per our hospital protocol to prevent VTE. Chemoprophylaxis is not routinely used unless the attending physicians consider it necessary.

The Caprini RAM is originally used for patients after surgery but it is also a convenient method to evaluate VTE risk just by calculating individual risk factors. In this model patients and procedure risk factors are assigned to different weighted points (ranging from 1 to 5) based on the risk of VTE for each factor. The Caprini score is calculated for individual patients based on different risk factors. A total risk factor score is calculated and patients are assorted into 5 risk level based on reported incidence of VTE: "very low risk" (0-2 points), "low risk" (3-4 points), "moderate risk" (5-6 points), "high risk" (7-8 points), and "very high risk" ( $>8$ points) leading to stratified prophylaxis. The distribution of patients according to the Caprini score are presented in Figure 1. The predominant stratified group have a Caprini score of 7 to 8 ( $n=2,661$, one patient with VTE), followed by the score 3 to 4 group ( $\mathrm{n}=90$, no patients with VTE), and the score 5 to 6 group (n=556, one patients with VTE).

\section{Results}

Of all 3,824 patients, 21 patients have VTE complications (Table 1) with an incidence of $0.55 \%$. Characteristics of patients, Caprine score, NHISS score, NEWS score, interval, treatment, 3-month follow-up of patients with VTE are listed in Table 2. The patients diagnosed with VTE included 17 males (80.95\%) and 4 female, with mean age of $67.95 \pm 14.99$ years (range, $49-89$ years). There are three patients who are diagnosed with PE and DVT. The interval is $8.10 \pm 1.44$ days (range, 6-12 days). Eleven patients with VTE complications showed complete patency with a minimum 90 days of follow-up. Five patients have post-thrombotic syndrome. One patient died among the 
Table 2 demographic and clinical characteristics of stroke patients with VTE

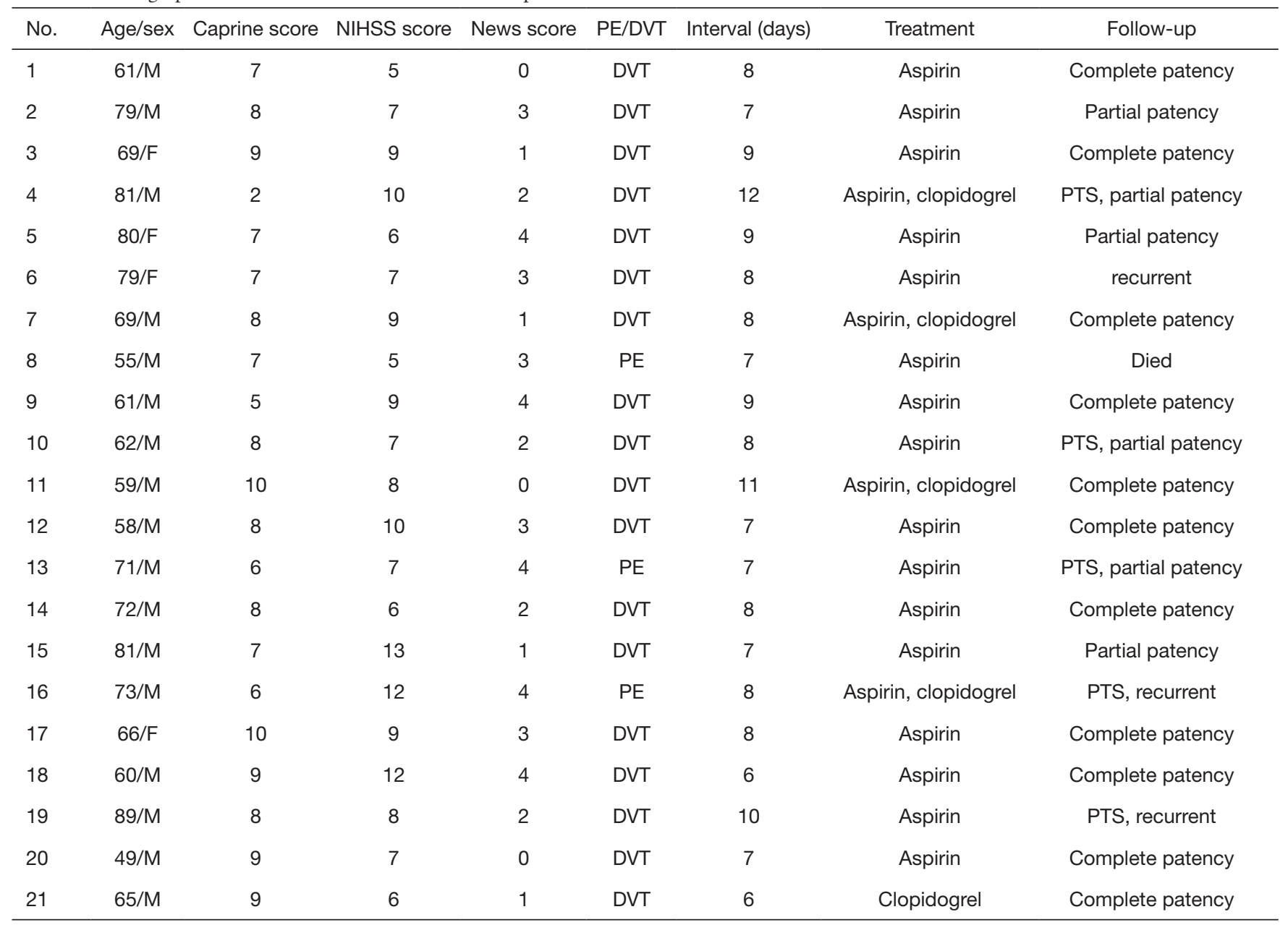

VTE, venous thromboembolism; DVT, deep vein thrombosis; PE, pulmonary embolism; PTS, post-thrombotic syndrome.

21 patients with VTE (Table 2).

As specified by the Caprini RAM, patients with a score of 3 and above belonged to moderate-to-high VTE risk group $(1,5)$, so mechanical and chemical prophylaxis are recommended to them. A total of 2,960 patients who don't have heparin contraindications received low-molecularweight heparin $(\mathrm{LMWH})$, and some of them developed VTE during hospitalizes period. In 26 patients who have high bleeding risk are prescribed mechanical prophylaxis instead of prophylactic anticoagulation.

Comparison of chemo thromboprophylaxis between patients with VTE group and those without VTE group are presented in Table 3. The mean age of VTE patients $(\mathrm{n}=21)$ is $67.95 \pm 14.99$ years. Medical co-morbidity (atrial fibrillation, diabetes, hyperbaric therapy) in patients with VTE are found to be significantly higher than non-VTE patients $(\mathrm{P}<0.05)$. The Caprini score in patients with VTE is significantly higher than non-VTE group $(8.05 \pm 2.38$ vs. $7.11 \pm 1.61$, respectively, $\mathrm{P}<0.01)$. The NIHSS score is also turned out to be obviously higher in patients with VTE $(7.71 \pm 5.98$ vs. $3.36 \pm 3.68$, respectively, $\mathrm{P}<0.05)$ than non-VTE group. D-dimer in patients with VTE is also significantly higher than non-VTE group $(5.28 \pm 6.86 v s$. $0.82 \pm 1.79$ respectively, $\mathrm{P}<0.01)$. No significant differences are found between the groups when compared age, hypertension, coronary heart disease, cancer, NEWS score, prothrombin time (PT), activated partial thromboplastin time (APTT), and thrombin time (TT) $(\mathrm{P}>0.05)$ (Table 3).

\section{Discussion}

There is substantial evidence that VTE is a common 
Table 3 comparisons between patients with VTE and those without VTE

\begin{tabular}{|c|c|c|c|}
\hline Characteristics & Patients with VTE $(n=21)$ & Patients without VTE $(n=3,803)$ & $P$ value \\
\hline Hypertension, n (\%) & $15(71.4)$ & $3,062(80.5)$ & 0.295 \\
\hline Atrial fibrillation, n (\%) & $5(23.8)$ & $356(9.4)$ & 0.024 \\
\hline Coronary heart disease, n (\%) & $1(4.8)$ & $362(9.5)$ & 0.458 \\
\hline Cancer, n (\%) & $0(0.0)$ & $50(1.3)$ & 0.597 \\
\hline Hyperbaric therapy, n (\%) & $11(52.4)$ & $665(17.5)$ & 0.000 \\
\hline NIHSS score, mean \pm SD & $7.71 \pm 5.98$ & $3.36 \pm 3.68$ & 0.000 \\
\hline NEWS score, mean \pm SD & $1.71 \pm 1.06$ & $1.54 \pm 1.11$ & 0.481 \\
\hline $\mathrm{TT}$, mean $\pm \mathrm{SD}$ & $18.11 \pm 1.37$ & $19.89 \pm 5.13$ & 0.120 \\
\hline D-dimer, mean \pm SD & $5.28 \pm 6.86$ & $0.82 \pm 1.79$ & 0.007 \\
\hline Caprini score, mean \pm SD & $8.05 \pm 2.38$ & $7.11 \pm 1.61$ & 0.008 \\
\hline
\end{tabular}

VTE, venous thromboembolism; NIHSS, National Institutes of Health Stroke Scale; NEWS, National Early Warning Score; PT, prothrombin time; APTT, activated partial thromboplastin time; TT, thrombin time; SD, standard deviation.

and frequent complication in stroke patients $(6,7)$, and if precautions are not taken then the incidence of VTE in patients with stroke goes up to $30-40 \%$ (8). Early identification and prevention of high risk VTE patients have critical significance to reduce the mortality and disability in stroke patients. Caprini is developed as a tool to evaluate DVT risk in patients after surgery, It consists of five parts and each with different factors (9).

In our study, we found the incidence of VTE in stroke is $0.55 \%(21 / 3,824)$, that is acceptable. The incidence in our study is lower than the study presented by Pannucci et al. that reported incidence rate of $0.97 \%$ (5). This study found that $80.95 \%$ of VTE occurred in males, which is supported by previous findings which reported men are more prone to VTEs than women (10). Three of 21 patients have a recurrence during the study, and the recurrence rate is $14.29 \%$. There's a lot of evidence to suggest that venous thrombosis has a high recurrence rate, especially PE. The main causes of thrombosis recurrence in stroke patients are vascular wall injury, slow blood flow and hypercoagulability $(11,12)$. The mean age of the patients in our study is $68.27 \pm 11.33$ years which is supported the previous conclusion.

Studies by different scholars have shown that early use of heparin can help to reduce the incidence of thrombotic events and mortality in stroke patients (13-15). The results of a study of 3,842 acute stroke patients in the United States (1) showed 2,960 (77.04\%) patients received VTE prophylaxis by using LMWH subcutaneously; 12 patient $(0.04 \%)$ developed thrombocytopenia and 6 patients $(0.02 \%)$ developed major bleeding. Although each anticoagulant carries risks still chemical prophylaxis should be used in patients at high risk of venous thrombosis with a principle that the benefits outweigh the risks (16). And that's why it's so important to identify high-risk groups. In clinical practice, it is necessary to timely assess whether the patient have complications or not according to their physical signs and laboratory indicators. PT and APTT are the most common and sensitive screening tests that reflect the coagulation system.

During the time of our study, 21 patients developed venous thrombosis, 8 in Caprini score of $>8$ group, 11 in Caprini score of >7-8 group, 1 in Caprini score of 5-6 group, and 1 in Caprini score of 0-2 group (Figure 1). Patients with a score of $>8$ belong to "super-high-risk" group of Caprini RAM; so for such patients extended (30 days) chemoprophylaxis should be used in addition with mechanical prophylaxis $(17,18)$. One patient with a Caprini score between 0 and 2 doesn't receive chemo prophylaxis and developed VTE. The patients 
with predisposing factors and Caprini score between 0 and 2 should be emphasized with early ambulation and mechanical prophylaxis.

In our study found that the patients with VTE complication have significantly higher $(\mathrm{P}<0.05)$ age, Caprini score, NIHSS score, NEWS score, PT, TT and D-Dimer than those without VTE complication (Table 3). The NIHSS score has been widely used in clinical prognosis assessment and various studies. The higher the score, the worse the prognosis, which is the same with the result of our study. A study shows that VTE, D-dimer is a beneficial biomarker for diagnosis of VTE in after stroke patients (19).

There are still some limitations in this study. It is a single center study. In addition, ultrasound imaging is used every time when there is a clinical suspicion; therefore, we do not check for asymptomatic VTE in stroke patients. The data in this study may be less than the actual incidence of VTE.

In conclusion, the Caprini score is highly recommended to assess the risk of venous thrombosis in stroke patients. Mechanical prophylaxis and chemical prophylaxis are recommended for patients.

\section{Acknowledgments}

The authors acknowledge Steven Zhang for checking the language of the manuscript. The authors also thank reviewers and editors for their inspiring and constructive suggestions.

Funding: None.

\section{Footnote}

Conflicts of Interest: All authors have completed the ICMJE uniform disclosure form (available at http://dx.doi. org/10.21037/apm.2020.04.20). The authors have no conflicts of interest to declare.

Ethical Statement: The authors are accountable for all aspects of the work in ensuring that questions related to the accuracy or integrity of any part of the work are appropriately investigated and resolved. The study is approved by National Ethics Committee of Shanghai Tenth People's Hospital of Tongji University (No. 2019-k-51). All the patients provided the written informed consent.

Open Access Statement: This is an Open Access article distributed in accordance with the Creative Commons Attribution-NonCommercial-NoDerivs 4.0 International
License (CC BY-NC-ND 4.0), which permits the noncommercial replication and distribution of the article with the strict proviso that no changes or edits are made and the original work is properly cited (including links to both the formal publication through the relevant DOI and the license). See: https://creativecommons.org/licenses/by-nc-nd/4.0/.

\section{References}

1. Douds GL, Hellkamp AS, Olson DM, et al. Venous thromboembolism in the Get With The Guidelines-Stroke acute ischemic stroke population: incidence and patterns of prophylaxis. J Stroke Cerebrovasc Dis 2014;23:123-9.

2. Kamphuisen PW, Agnelli G, Sebastianelli M. Prevention of venous thromboembolism after acute ischemic stroke. J Thromb Haemost 2005;3:1187-94.

3. Hillbom M, Erila T, Sotaniemi K, et al. Enoxaparin vs heparin for prevention of deep-vein thrombosis in acute ischaemic stroke: a randomized, double-blind study. Acta Neurol Scand 2002;106:84-92.

4. Stroud W, Whitworth JM, Miklic M, et al. Validation of a venous thromboembolism risk assessment model in gynecologic oncology. Gynecol Oncol 2014;134:160-3.

5. Pannucci CJ, Osborne NH, Wahl WL. Creation and validation of a simple venous thromboembolism risk scoring tool for thermally injured patients: analysis of the National Burn Repository. J Burn Care Res 2012;33:20-5.

6. Horsted F, West J, Grainge MJ. Risk of venous thromboembolism in patients with cancer: a systematic review and meta-analysis. PLoS Med 2012;9:e1001275.

7. Field TS, Hill MD. Prevention of deep vein thrombosis and pulmonary embolism in patients with stroke. Clin Appl Thromb Hemost 2012;18:5-19.

8. Turpie AG. Prophylaxis of venous thromboembolism in stroke patients. Semin Thromb Hemost 1997;23:155-7.

9. Barbar S, Noventa F, Rossetto V, et al. A risk assessment model for the identification of hospitalized medical patients at risk for venous thromboembolism: the Padua Prediction Score. J Thromb Haemost 2010;8:2450-7.

10. Kakkos S, Tsolakis I, Katsamouris A, et al. Risk stratification approaches for venous thromboembolism (VTE) prophylaxis in surgical patients. Hellenic J Surg 2013;85:18-27.

11. Heit JA, Mohr DN, Silverstein MD, et al. Predictors of recurrence after deep vein thrombosis and pulmonary embolism: a population-based cohort study. Arch Intern Med 2000;160:761-8.

12. Maufus M, Elias A, Barrellier MT, et al. Diagnosis of deep 
vein thrombosis recurrence: Ultrasound criteria. Thromb Res 2018;161:78-83.

13. Kay R, Wong KS, Yu YL, et al. Low-molecular-weight heparin for the treatment of acute ischemic stroke. N Engl J Med 1995;333:1588-93.

14. Yusuf S, Mehta SR, Xie C, et al. Effects of reviparin, a low-molecular-weight heparin, on mortality, reinfarction, and strokes in patients with acute myocardial infarction presenting with ST-segment elevation. JAMA 2005;293:427-35.

15. Winningham MJ, Haussen DC, Nogueira RG, et al. Periprocedural heparin use in acute ischemic stroke endovascular therapy: the TREVO 2 trial. J Neurointerv Surg 2018;10:611-4.

16. Pollak U. Heparin-induced thrombocytopenia

Cite this article as: Zhu X, Zhang T, Zhou L, Yin X, Dong Q. Stratification of venous thromboembolism risk in stroke patients by Caprini score. Ann Palliat Med 2020;9(3):631-636. doi: 10.21037/apm.2020.04.20 complicating extracorporeal membrane oxygenation support in pediatric patients: review of the literature and alternative anticoagulants. Perfusion 2018;33:7-17.

17. Adams HP Jr, Adams RJ, Brott T, et al. Guidelines for the early management of patients with ischemic stroke: A scientific statement from the Stroke Council of the American Stroke Association. Stroke 2003;34:1056-83.

18. Leys D, Bogousslavsky J, Bath P, et al. EUSI Executive Committee, EUSI Writing Committee. Prevention. European Stroke Initiative. Cerebrovasc Dis 2004;17:15-29.

19. Zhang D, Li F, Du X, et al. Diagnostic accuracy of biomarker D-dimer in patients after stroke suspected from venous thromboembolism: A diagnostic meta-analysis. Clin Biochem 2019;63:126-34. 